\title{
Feedback Control of Impact Dynamics: the Bouncing Ball Revisited
}

\author{
Renaud Ronsse and Rodolphe Sepulchre
}

\begin{abstract}
We study the the design of a tracking controller for the popular bouncing ball model: the continuous-time actuation of a table is used to control the impacts of the table with a bouncing ball. The proposed control law uses the impact times as the sole feedback information. We show that the acceleration of the table at impact plays no role in the stability analysis but is an important parameter for the robustness of the feedback system to model uncertainty, in particular to the uncertainty on the coefficient of restitution.
\end{abstract}

\section{INTRODUCTION}

A broad set of rhythmic tasks are routinely accomplished by humans and animals. The particular issue investigated in this paper relates to the question of feedback: how much feedback is needed to perform a rhythmic task and how is this feedback processed to produce control actions ? This question reaches across several disciplines. In neuroscience, it has been shown that humans recruit distinct brain structures to control rhythmic tasks as opposed to discrete tasks (reaching, grasping, pointing) [1]. In robotics, Buehler, Koditschek and Kindlmann [2], [3], [4] were the first to investigate the role of feedback in a rhythmic setup.

Impact tasks are particular rhythmic tasks where a "robot" controls one or several "object(s)" through periodic impacts. The resulting dynamics are hybrid because they couple the continuous-time dynamics of the uncontrolled object and the discrete impact dynamics. Impact control systems involving ball(s) and robot(s) are also referred as juggling systems [4], [5], [6], [7].

The benchmark impact dynamics studied in the present paper is the popular 1-D Bouncing Ball: a ball vertically bounces on a moving table. This impact system is instrumental in capturing in a simple way the central features of more complicated examples (see e.g. [7], [8]). The Bouncing Ball has been first analyzed for its rich dynamical behavior when the table follows a simple sinusoidal motion [9], [10], [11]. The amplitude and the frequency of the sinusoid can be tuned to stabilize periodic patterns. The actuation is in this case sensorless because it requires no feedback at all. Sensorless strategies have been generalized to a 2-D juggling device, with successful experimental validation [12], [13]. Feedback control algorithms have also been proposed for the Bouncing Ball, including the mirror law algorithms developed in [2], [3], [4]. Thereafter, the Bouncing Ball has been used as a benchmark to study controllability and stabilization of impact dynamics [14], [15], [5], [16], [6] in 1-D or 2-D environment,

R. Ronsse and R. Sepulchre are with the Department of Electrical Engineering and Computer Science (Montefiore Institute), Université de Liège, 4000 Liège, Belgium [R.Ronsse, R.Sepulchre]@ulg.ac.be with successful experimental validations. These questions have been generalized to other impact systems [17], [7], [18], [8], [19].

The continuous-time control strategy presented in this paper is an actuation of the table, reinitialized after each impact, based on the sole information of the impact times. It achieves dead-beat convergence of the discrete state of the system (the impact position and the (post-)impact velocity of the ball) to any admissible reference trajectory. The parametrization of the continuous-time control law as a piecewise quadratic function of time is used to emphasize the role of the impact acceleration in the robustness of the control system. While the impact acceleration plays no role in the stability analysis of the nominal model, we show that a well-chosen negative value of the acceleration eliminates the static error due to an uncertain coefficient of restitution. Negative values of the acceleration are also favorable to the stability of the perturbed system. The advantage of a negative impact acceleration is discussed in the broader perspective of the sensing requirements of a given control law.

The rest of this paper is organized as follows. In Section II, the derivation of the 1-D Bouncing Ball model is reviewed. In Section III, the piecewise quadratic control law is presented. In particular, Section III-C illustrates how this control law compares to the sensorless sinusoidal trajectory [9], [10], [11] and the mirror law [2], [3], [4]. Section IV generalizes the controller to output feedback, where the measured output is the sequence of impact times. In Section V, we discuss the role of the impact acceleration with respect to the robustness

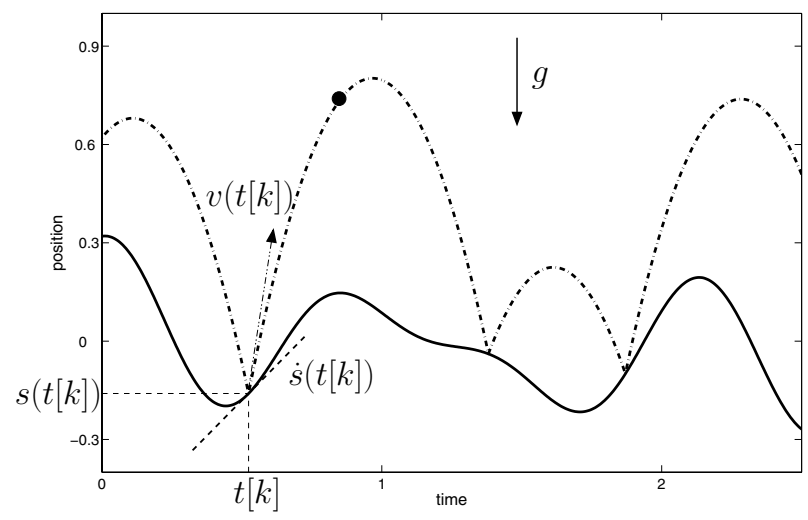

Fig. 1. 1-D Bouncing Ball. The table (ball, resp.) trajectory is depicted with solid (dash-dotted, resp.) lines over time. At time $t[k]$ ( $k^{\text {th }}$ impact), the table (and ball) position is $s(t[k])$, the table velocity is $\dot{s}(t[k])$ and the ball post-impact velocity is $v(t[k])$. 
of the feedback system in the presence of an uncertain coefficient of resitution. The paper ends with a discussion.

\section{BOUNCING BALL MODEL}

This section reviews the mathematical model of a ball that vertically bounces on a moving table. It is one of the simplest examples of impact dynamics: the table motion is the continuous-time actuation $s(t)$ while the ball dynamics are only governed by the gravitational field $g$ and the impacts with the table. The Bouncing Ball model has been first studied in [9] and [10].

Between the $k^{\text {th }}$ and the $(k+1)^{\text {th }}$ impacts, occurring at time $t[k]$ and $t[k+1]$, the ball follows a parabolic trajectory, see Figure 1. The table (and ball) impact position therefore obeys the following discrete-time dynamics:

$$
\begin{array}{r}
s(t[k+1])=s(t[k])+v(t[k])(t[k+1])-t[k]) \\
\left.-\frac{g}{2}(t[k+1])-t[k]\right)^{2}
\end{array}
$$

where $v(t[k])$ is the post-impact velocity and $g$ the gravitational field. Similarly the pre-impact velocity, $v^{-}(t[k+1])$ is equal to:

$$
v^{-}(t[k+1])=v(t[k])-g(t[k+1]-t[k])
$$

Based on Newton's law, the relative velocity of the ball with respect to the table is reversed at impact, up to a coefficient of restitution $0 \leq e \leq 1$ that models the energy dissipation:

$$
v(t[k+1])-\dot{s}(t[k+1])=-e\left(v^{-}(t[k+1])-\dot{s}(t[k+1])\right)
$$

Equation (3) assumes that the table motion is unaffected by the impacts. This assumption is valid if the table is largely heavier than the ball.

The complete Bouncing Ball dynamics are described by the discrete Poincare map, whose state is the impact position $s[k]=s(t[k])$ and post- velocity $v[k]=v(t[k])$ :

$$
\begin{array}{cc}
s[k+1]= & s[k]+v[k](t[k+1])-t[k]) \\
\left.-\frac{g}{2}(t[k+1])-t[k]\right)^{2} \\
v[k+1]= & -e v[k]+e g(t[k+1]-t[k]) \\
& +(1+e) \dot{s}[k+1]
\end{array}
$$

where $\dot{s}[k]=\dot{s}(t[k])$. Equation (5) is derived from (2) and (3).

The objective of the paper is to design a continuous-time motion $s(t)$ that controls the ball to track a discrete-time reference trajectory $\left(s_{\rho}[k], v_{\rho}[k]\right)$, impact after impact.

\section{CONTROL PARAMETRIZATION}

\section{A. State feedback dead-beat control}

The control law proposed in this paper is a piecewise quadratic motion of the table, re-initialized after each impact:

$s(t)=s_{u}[k+1]+\dot{s}_{u}[k+1]\left(t-t_{u}[k+1]\right)+\frac{\gamma}{2}\left(t-t_{u}[k+1]\right)^{2}$
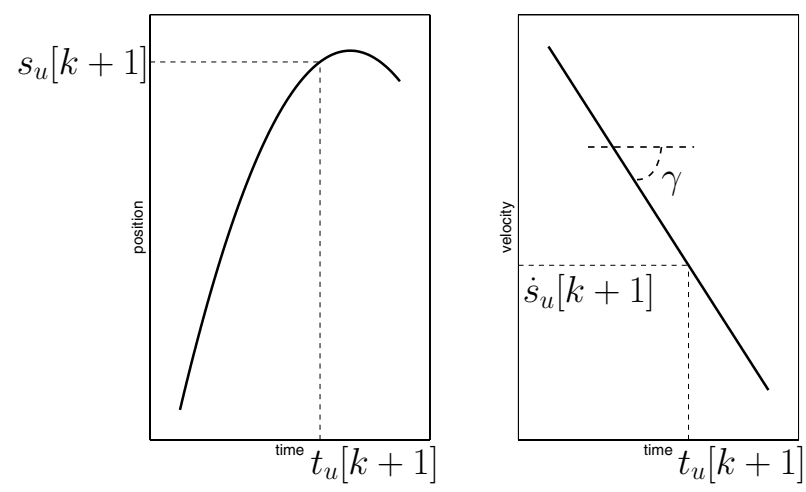

Fig. 2. Local position (left) and velocity (right) profiles of the control trajectory. The position profile is given by (6) while the velocity is its first derivative. $\gamma$ is the acceleration.

for $t[k]<t \leq t[k+1], \gamma$ denoting the table acceleration. At time $t=t_{u}[k+1]$, the table position (velocity, resp.) is equal to $s_{u}[k+1]\left(\dot{s}_{u}[k+1]\right.$, resp.), see Figure 2 .

We choose a quadratic control parametrization because the expression of impact times remains analytical in that case. The impact time $t[k+1]$ is indeed the solution of (4) and (6) at time $t=t[k+1]$, which defines a a second order polynomial in $t$. Its solution and the reference trajectory $\left(s_{\rho}, v_{\rho}\right)$ determine the tracking controller

$$
\begin{aligned}
t_{u}[k+1] & =t[k]+\frac{v[k]+\sqrt{v[k]^{2}-2 g\left(s_{\rho}[k+1]-s[k]\right)}}{g} \\
s_{u}[k+1] & =s_{\rho}[k+1] \\
\dot{s}_{u}[k+1] & =\frac{v_{\rho}[k+1]-e \sqrt{v[k]^{2}-2 g\left(s_{\rho}[k+1]-s[k]\right)}}{1+e}
\end{aligned}
$$

as a function of the state $(s[k], v[k])$ and impact time $t[k]$. Dead-beat convergence of this tracking control law, that is, convergence of the solution to the reference trajectory after a finite number of time steps, is established in the next section.

\section{B. Dead-beat convergence}

Substituting (7), (8) and (9) into (6) at time $t=t[k+1]$, we find:

$$
\begin{aligned}
& s[k+1]= s_{\rho}[k+1] \\
&+\frac{v_{\rho}[k+1]-e \sqrt{v[k]^{2}-2 g\left(s_{\rho}[k+1]-s[k]\right)}}{1+e} \\
&\left(t[k+1]-t[k]-\frac{v[k]+\sqrt{v[k]^{2}-2 g\left(s_{\rho}[k+1]-s[k]\right)}}{g}\right) \\
&+\frac{\gamma}{2}\left(t[k+1]-t[k]-\frac{v[k]+\sqrt{v[k]^{2}-2 g\left(s_{\rho}[k+1]-s[k]\right)}}{g}\right)^{2}
\end{aligned}
$$

The flight time is equal to (see (4)):

$$
t[k+1]-t[k]=\frac{v[k]+\sqrt{v[k]^{2}-2 g(s[k+1]-s[k])}}{g}
$$

Substituting (11) into (10), we see that the positive root of this equation is simply:

$$
s[k+1]=s_{\rho}[k+1]
$$


and subsequently:

$$
\begin{aligned}
v[k+1] & =v_{\rho}[k+1] \\
t[k+1] & =t_{u}[k+1]
\end{aligned}
$$

To summarize, the continuous-time control law determined by the quadratic expression (6) and the discrete control (7), (8) and (9), ensures dead-beat convergence of the impact state after one time step. The acceleration $\gamma$ in (6) is a free parameter and does not influence the convergence.

\section{Two popular control schemes}

In this section, we review two popular techniques to stabilize a period-one motion of the Bouncing Ball. A period-one motion is characterized by a constant reference at impacts: $\left(s_{\rho}[k], v_{\rho}[k]\right)=\left(s_{\rho}^{\star}, v_{\rho}^{\star}\right), \forall k$.

The first technique does not require any sensory feedback: the control law is the sinusoid $s(t)=A \sin (\omega t)$. This is the input considered in [9] and [10]. The resulting dynamics is unexpectedly rich since the ball obeys a series of period doubling and complex bifurcations, from period-one to chaos, as the amplitude parameter $A$ increases. In particular, the period-one motion is stable if [11]:

$$
\pi n \frac{1-e}{1+e}<\frac{A \omega^{2}}{g}<\sqrt{\pi^{2} n^{2}\left(\frac{1-e}{1+e}\right)^{2}+\frac{4\left(1+e^{2}\right)^{2}}{(1+e)^{4}}}
$$

where $n=1$ when there is one table period between two successive impacts. Steady-state ball impact position and post-impact velocity are given by:

$$
\begin{aligned}
s_{\rho}^{\star} & =A \sin \left(\arccos \left(\frac{(1-e) \pi n g}{(1+e) A \omega^{2}}\right)\right) \\
& =A \sqrt{1-\left(\frac{(1-e) \pi n g}{(1+e) A \omega^{2}}\right)^{2}} \\
v_{\rho}^{\star} & =\frac{\pi n g}{\omega}
\end{aligned}
$$

The amplitude $A$ and frequency $\omega$ can be tuned to match any $\left(s_{\rho}^{\star}, v_{\rho}^{\star}\right)$. It can be shown that, when the stability condition (15) is fulfilled, the table acceleration at impact is always negative (see Figure 3, top).

In contrast to the sensorless sinusoid, the mirror law [2], [3], [4] requires a permanent tracking of the ball, since the table mirrors the ball trajectory:

$$
s(t)=\left(\frac{-(1-e)}{1+e}-\kappa_{1}\left(E_{\rho}^{\star}-E[k]\right)\right) \beta(t)
$$

where $\beta(t)$ denote the ball position at time $t$. The second term in (18) is a proportional feedback on the ball energy that is used to isolate a particular reference $\left(s_{\rho}^{\star}, v_{\rho}^{\star}\right)$, and vanishes in steady-state. In steady-state, the mirror law behavior is depicted in Figure 3, bottom.

Comparing the sinusoidal law and the mirror law in Figure 3 , we see that both of them stabilize the Bouncing Ball period-one at the same impact position and post-impact velocity, but with significant differences in the underlying continuous-time control law. Matching our piecewise quadratic control parametrization with these two steady-state
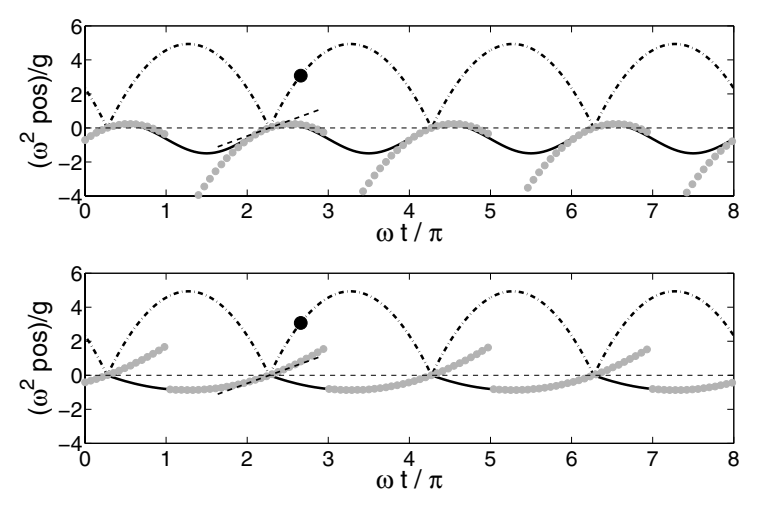

Fig. 3. Comparison of the sinusoidal trajectory (top) and the mirror law (bottom) to stabilize the period-one pattern. The table (ball, resp.) adimensional position is depicted with solid (dash-dotted, resp.) lines over adimensional time. The grey dots denote the piecewise quadratic trajectory that match the table position, velocity and acceleration at impact.

control laws (grey dots in Figure 3), we observe that the sinusoidal trajectory is decelerating at impact $(\gamma<0)$ while the mirror law is accelerating: a simple calculation shows that the acceleration parameter of the steady-state mirror law is $\gamma=\frac{1-e}{1+e} g>0$.

The two control strategies that stabilize the same pattern are thus clearly distinct. We emphasize that the first one is sensorless while the second requires a permanent tracking of the ball, which suggests that the acceleration at impact possibly influences the feedback requirements of the control law.

\section{OUTPUT FEEDBACK DEAD-BEAT CONTROL}

The mirror law described in the previous section is based on a continuous-time tracking of the ball, and the sensing requirements of such a control law can be challenging. Likewise, the piecewise quadratic control proposed in Section III uses the discrete state $(s[k], v[k])$.

To reduce the sensing requirements of the controller, we will now assume that only the table motion is measured, together with the impact times $t[k]$. Impact times are easily measured (by an accelerometer recording the table vibrations, a microphone, ...). They provide the impact position $s[k]$ and velocity $\dot{s}[k]$ from the continuous-time measurement $s(t)$. In this section, we present a dead-beat observer that reconstructs the post-impact ball velocity $v[k]$ from the measured impact times $t[k]$ and table motion $s(t)$.

Post-impact velocity is estimated by an observer that is a copy of the velocity dynamics (5):

$$
\hat{v}[k]=-e v[k-1]+e g(t[k]-t[k-1])+(1+e) \dot{s}[k]
$$


while $v[k-1]$ is obtained from (4):

$$
\begin{array}{r}
\hat{v}[k]=-e\left(\frac{s[k]-s[k-1]}{t[k]-t[k-1]}+\frac{g}{2}(t[k]-t[k-1])\right) \\
+e g(t[k]-t[k-1])+(1+e) \dot{s}[k] \\
=e \frac{g}{2}(t[k]-t[k-1])-e \frac{s[k]-s[k-1]}{t[k]-t[k-1]} \\
+(1+e) \dot{s}[k]
\end{array}
$$

Equation (20) is a dead-beat velocity observer using only the impact times as sole input in addition to the table motion. Dead-beat convergence is ensured in one time-step since $\hat{v}[k]=v[k], \forall k>1$.

Dead-beat convergence of the output feedback controller obtained by replacing $v[k]$ by $\hat{v}[k]$ in (7), (8) and (9) is also straightforwardly established since $\hat{v}[k]=v[k], \forall k>1$. The dead-beat convergence of the output feedback controller is in two steps: one impact is required for the observer convergence and another one for the controller convergence.

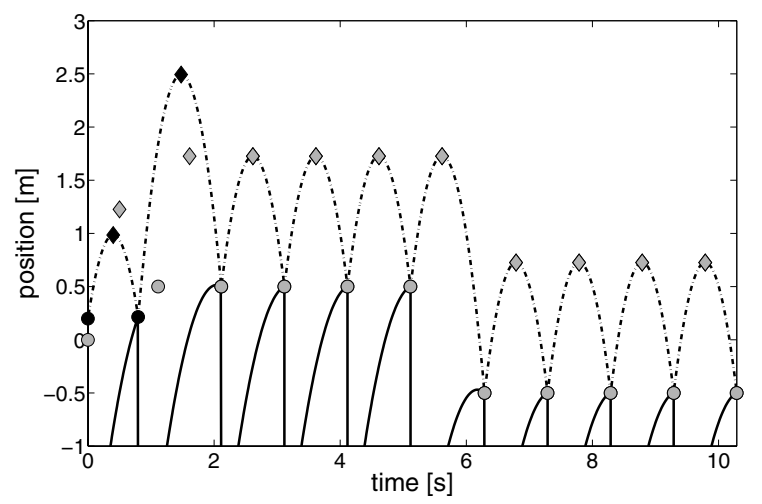

Fig. 4. Output feedback control of the Bouncing Ball with piecewise quadratic trajectory. The table (ball, resp.) position is depicted with solid (dash-dotted, resp.) lines over time. Actual impacts position (apex position, resp.) are denoted with black circles (black diamonds, resp.). Reference positions are accordingly denoted with grey markers. $e=0.9, \gamma<0$.

The dead-beat convergence is illustrated in Figure 4. At the third impact, both the reference impact position and the reference apex (which is an image of the reference postimpact velocity) are reached. The same figure illustrates the proper tracking of time-varying references since both the position and velocity references change at the seventh impact.

\section{ROBUSTNESS TO COEFFICIENT OF RESTITUTION}

So far, the acceleration parameter $\gamma$ appearing in (6) was considered as a free parameter of the control law that plays no role in the convergence analysis. This section stresses the importance of this parameter for robustness purposes. We show that a particular negative acceleration is able to efficiently reject the perturbations induced by a poor estimate of the coefficient of restitution $e$.

The velocity observer (20) and the control input (9) assume an accurate knowledge of the coefficient of restitution $e$. However, in a real experimental setup, this parameter is hard to estimate, and varies with respect to the experimental conditions. Suppose now that the actual coefficient of restitution varies impact after impact, so that (5) becomes:

$v[k+1]-\dot{s}[k+1]=-(e+\Delta e[k+1])\left(v^{-}[k+1]-\dot{s}[k+1]\right)$

where $\Delta e[k+1]$ models the variation of the coefficient of restitution at time $t[k+1]$, w.r.t. the estimated value $e$. For the sake of simplicity, we will only address the steady-state system behavior - i.e. by considering a constant reference $\left(s_{\rho}^{\star}, v_{\rho}^{\star}\right)$ - of the linearized feedback system, as derived in the Appendix.

From the linear state-space representation (29), we find the following closed-loop input-to-state transfer functions:

$$
\begin{aligned}
S(z) & =S_{\rho}(z)+\frac{2(1-e)}{1+e} \frac{1}{z} E(z) \\
V(z) & =V_{\rho}(z)+\frac{2}{1+e} \frac{z+\left(e^{2}+\frac{\gamma}{g}(1+e)^{2}\right)}{z} E(z)
\end{aligned}
$$

where $S(z), V(z), S_{\rho}(z), V_{\rho}(z)$ and $E(z)$ refer to the $z$-transforms of $g \delta s[k] /\left(v_{\rho}^{\star}\right)^{2}, \delta v[k] / v_{\rho}^{\star}, g \delta s_{\rho}[k] /\left(v_{\rho}^{\star}\right)^{2}$, $\delta v_{\rho}[k] / v_{\rho}^{\star}$ and $\Delta e[k]$, respectively. In (22) and (23), the absence of dynamics in the transfer from references to states is due to the deadbeat convergence established in Section IV.

From (23), we see that the acceleration $\gamma$ can be designed to place the zero of the transfer function from $E(z)$ to $V(z)$. That design parameter will be discussed to optimize either the static or the dynamic performance.

\section{A. Static performance}

To let the post-impact velocity converge toward the reference $v_{\rho}[k]$, assuming a constant perturbation $\Delta e[k]=\Delta e$, one has to cancel the static gain of the transfer function from $E(z)$ to $V(z)$. This amounts to place the zero of (23) at $z=-1$, which requires the following acceleration:

$$
\gamma_{\text {stat. }}=-\frac{1+e^{2}}{(1+e)^{2}} g
$$

Interestingly, this optimal acceleration depends only on $e$, that is, the estimated coefficient of restitution. Figure 5 illustrates the behavior of the feedback system when the coefficient of restitution is estimated at $e=0.9$ while the real one is only $e+\Delta e=0.7$. The desired post-impact velocity is reached because the difference between impact and apex positions is the same for the reference as for the actual trajectory. It should be noticed, however, that a static error persists on the reference position: the static gain from $E(z)$ to $S(z)$ is indeed independent of $\gamma$ (see (22)). This static error does not appear to be detrimental to the robustness of the feedback system.

It is of interest to relate the particular acceleration identified in (24) to the sinusoidal control discussed in Section III-C. The period-one stability condition (15) defines the 


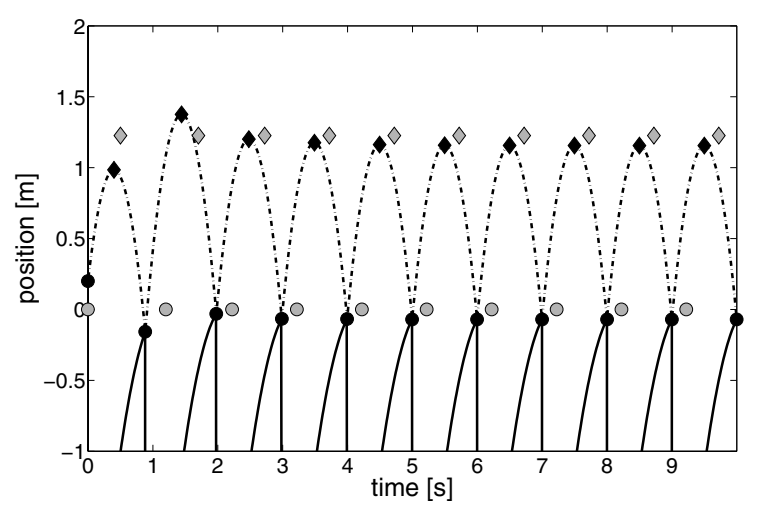

Fig. 5. Output feedback control of the Bouncing Ball with piecewise quadratic trajectory. The table (ball, resp.) position is depicted with solid (dash-dotted, resp.) lines over time. Actual impacts position (apex position, resp.) are denoted with black circles (black diamonds, resp.). Reference positions are accordingly denoted with grey markers. $e+\Delta e=0.9-0.2$, $\gamma$ is given by (24).

following steady-state acceleration range:

$$
\frac{-2\left(1+e^{2}\right)}{(1+e)^{2}} g<\ddot{s}^{\star}<0
$$

The optimal acceleration (24) is therefore exactly the middle point of this range.

\section{B. Dynamic performance}

Robustness to a static error on $e$ is not the primary issue in real experiments because the average value of $e$ is easy to determine. In contrast, robustness is required against the sustained variability of $e$. By placing the zero of (23) at $z=0$, the dynamics from $E(z)$ to $V(z)$ are exactly canceled:

$$
\gamma_{\text {dyn. }}=-\frac{e^{2}}{(1+e)^{2}} g
$$

Either the static tuning, or the dynamic tuning, resulted in negative acceleration at impact, with

$$
\gamma_{\text {stat. }}<\gamma_{\text {dyn }} \leq 0
$$

\section{DISCUSSION}

This paper addressed the control of impact systems by means of the popular example of the 1-D Bouncing Ball [9], [10]. We designed a piecewise quadratic trajectory whose parameters are reinitialized at each impact based on the desired reference trajectory and the current impact state. Post-impact velocity is reconstructed by a dead-beat observer that only uses the continuous-time measurement of the table $s(t)$ and the impact times $t[k]$. This controller is dead-beat since the reference state is reached for $k>2$.

The impact acceleration $\gamma$ was shown to be a free parameter of the control law. Section V illustrates how a proper tuning of this parameter can improve the robustness of the system, by rejecting the perturbations that are due to a bad estimate of the coefficient of restitution. Two particular negative accelerations were examined: the first one $\left(\gamma_{\text {stat. }}\right)$ was shown to eliminate the static error of the impact velocity and, more interestingly, the second one $\left(\gamma_{\text {dyn. }}\right)$ canceled the dynamics between a varying coefficient of restitution and the impact velocity.

A link was established between $\gamma_{\text {stat. }}$ and the stability range of the period-one pattern with sinusoidal actuation [11]. We hypothesize that the negative value of the acceleration impact associated to sensorless control is responsible for the good results that have been obtained experimentally (see e.g. [13] for an adaptation to a 2-D impact system). In contrast, the mirror law algorithm [2], [3], [4], which requires a continuous tracking of the ball was shown to cause positive acceleration of the table at impacts because it mirrors the gravity. An observer-based controller based on this strategy is expected to be non robust to uncertainty on the coefficient of restitution [8]. More generally, we expect control laws with positive impact acceleration to require more demanding sensing capabilities. When human subjects are asked to bounce a ball with a racket, it has been shown that they impact the ball with a negative acceleration belonging to the sensorless stability range [20], [21], i.e. close to the optimal values identified in (24) and (26).

In summary, the piecewise quadratic continuous-time control law presented in this paper provides a good insight to the success and limitations of several control schemes referenced in the literature. In particular, exploiting the local behavior of the sensorless sinusoidal actuation seems to be relevant in designing robust closed-loop controller requiring minimum sensory feedback.

An extended version of the present paper extends the proposed approach to the control of a two-dimensional juggling device [22]. In accordance with the discussion above, the proper tuning of the impact acceleration for robustness against the uncertainty of the impact model is shown to have a dramatic influence on the validation of the design in the lab.

\section{APPENDIX}

The linearized dynamics of the perturbed 1D bouncing ball dynamics (4) and (21), and the piecewise quadratic law (6) are given at the top of the next page (28), where $\delta e[k+1]$ is the small perturbation on the coefficient of restitution and is considered as an additional input. Using non-dimensional state variables, one obtains the state-space model (29), also given at the top of the next page. The state variables are small perturbations of the ball impact position $g \delta s[k+1] /\left(v_{\rho}^{\star}\right)^{2}$ and velocity $\delta v[k+1] / v_{\rho}^{\star}$; the impact time $g \delta t[k+1] / v_{\rho}^{\star}$ and the observed velocity $\delta \hat{v}[k+1] / v_{\rho}^{\star}$.

The matrix $\mathbf{A}^{\prime}$ is singular. This is a consequence of deadbeat convergence of the 1D bouncing ball, controlled with the piecewise quadratic law (6).

\section{ACKNOWLEDGMENTS}

This work was supported by the Belgian Program on Interuniversity Attraction Poles initiated by the Belgian Federal Science Policy Office. 


$$
\begin{aligned}
& \delta s[k+1]=\delta s[k]+\frac{2 \pi n}{\omega} \delta v[k]-\frac{\pi n g}{\omega}(\delta t[k+1]-\delta t[k]) \\
& \delta v[k+1]=-e \delta v[k]+e g(\delta t[k+1]-\delta t[k])+(1+e) \delta \dot{s}[k+1]+\frac{2}{1+e} \frac{\pi n g}{\omega} \delta e[k+1] \\
& \delta s[k+1]=\frac{2 e}{1+e} \delta s_{\rho}[k+1]+\frac{1-e}{1+e} \delta s[k]+\frac{1-e}{1+e} \frac{\pi n g}{\omega}(\delta t[k+1]-\delta t[k])-\frac{1-e}{1+e} \frac{2 \pi n}{\omega} \delta \hat{v}[k] \\
& \delta \dot{s}[k+1]=\frac{1}{1+e} \delta v_{\rho}[k+1]-\left(\frac{e}{1+e}+\frac{2 \gamma}{g}\right) \delta \hat{v}[k]+\left(\frac{e}{1+e}+\frac{\gamma}{g}\right) \frac{\omega}{\pi n}\left(\delta s_{\rho}[k+1]-\delta s[k]\right)+\gamma(\delta t[k+1]-\delta t[k]) \\
& \delta \hat{v}[k+1]=e \frac{g}{2}(\delta t[k+1]-\delta t[k])-e \frac{\omega}{2 \pi n}(\delta s[k+1]-\delta s[k])+(1+e) \delta \dot{s}[k+1] \\
& \left(\begin{array}{c}
\frac{g \delta s[k+1]}{\left(v_{\rho}^{\star}\right)^{2}} \\
\frac{\delta v[k+1]}{v_{\rho}^{\star}} \\
\frac{g \delta t[k+1]}{v_{\rho}^{\star}} \\
\frac{\delta \hat{v}[k+1]}{v_{\rho}^{\star}}
\end{array}\right)=\underbrace{\left(\begin{array}{cccc}
0 & 1-e & 0 & -(1-e) \\
0 & e^{2}+\frac{\gamma}{g}(1+e)^{2} & 0 & -\left(e^{2}+\frac{\gamma}{g}(1+e)^{2}\right) \\
1 & 1+e & 1 & 1-e \\
0 & e^{2}+\frac{\gamma}{g}(1+e)^{2} & 0 & -\left(e^{2}+\frac{\gamma}{g}(1+e)^{2}\right)
\end{array}\right)}_{\mathbf{A}^{\prime}}\left(\begin{array}{c}
\frac{g \delta s[k]}{\left(v_{\rho}^{\star}\right)^{2}} \\
\frac{\delta v[k]}{v_{\rho}^{\star}} \\
\frac{g \delta[k]}{v_{\rho}^{\star}} \\
\frac{\delta \hat{v}[k]}{v_{\rho}^{\star}}
\end{array}\right)+\underbrace{\left(\begin{array}{ccc}
1 & 0 & 0 \\
0 & 1 & \frac{2}{1+e} \\
-1 & 0 & 0 \\
0 & 1 & 0
\end{array}\right)}_{\mathbf{B}^{\prime}}\left(\begin{array}{c}
\frac{g \delta s_{\rho}[k+1]}{\left(v_{\rho}^{\star}\right)^{2}} \\
\frac{\delta v_{\rho}[k+1]}{v_{\rho}^{\star}} \\
\delta e[k+1]
\end{array}\right)
\end{aligned}
$$

\section{REFERENCES}

[1] S. Schaal, D. Sternad, R. Osu, and M. Kawato, "Rhythmic arm movement is not discrete," Nat Neurosci, vol. 7, no. 10, pp. 113643, 2004.

[2] M. Buehler, D. Koditschek, and P. Kindlmann, "A one degree of freedom juggler in a two degree of freedom environment," in IEEE/RSJ Conf. intelligent Systems and Robots, Tokyo, Japan, 1988, pp. pp. 9197.

[3] _ - "A family of robot control strategies for intermittent dynamical environments," IEEE Control Systems Magazine, vol. 10, no. 2, pp. pp. 16-22, 1990.

[4] - "Planning and control of robotic juggling and catching tasks," International Journal of Robotics Research, vol. 13, no. 2, pp. 101118, 1994.

[5] K. M. Lynch and C. K. Black, "Recurrence, controllability, and stabilization of juggling," IEEE Transactions on Robotics and Automation, vol. 17, no. 2, pp. 113-124, 2001.

[6] A. Zavala-Rio and B. Brogliato, "Direct adaptive control design for one-degree-of-freedom complementary-slackness jugglers," Automatica, vol. 37, no. 7, pp. 1117-1123, 2001.

[7] R. Sepulchre and M. Gerard, "Stabilization of periodic orbits in a wedge billiard," in 42nd IEEE Conference on Decision and Control, D. A. Lawrence and T. Parisini, Eds. Hawaii: IEEE, 2003, pp. 15681573.

[8] M. Gerard and R. Sepulchre, "Rhythmic stabilization of periodic orbits in a wedge," in IFAC World Congress, P. Horacek, M. Simandl, and P. Zitek, Eds., Prague, Czech Republic, 2005.

[9] P. J. Holmes, "The dynamics of repeated impacts with a sinusoidally vibrating table," J. Sound Vibration, vol. 84, no. 2, pp. 173-189, 1982.

[10] J. Guckenheimer and P. J. Holmes, Nonlinear oscillations, dynamical systems and bifurcations of vector fields. New York: Springer-Verlag, 1986.

[11] C. Bapat, S. Sankar, and N. Popplewell, "Repeated impacts on a sinusoidally vibrating table," J. Sound Vibration, vol. 108, no. 1, pp. 99-115, 1986.

[12] S. Schaal and C. G. Atkeson, "Open loop stable control strategies for robot juggling," in IEEE International Conference on Robotics and Automation, vol. 3, 1993, pp. 913-918.

[13] R. Ronsse, P. Lefevre, and R. Sepulchre, "Sensorless stabilization of bounce juggling," IEEE Transactions on Robotics, vol. 22, no. 1, pp. 147-159, 2006.

[14] B. Brogliato and A. Zavala-Rio, "On the control of complementaryslackness juggling mechanical systems," IEEE Transactions on Automatic Control, vol. 45, no. 2, pp. 235-246, 2000.

[15] T. L. Vincent and A. I. Mees, "Controlling a bouncing ball," International Journal of Bifurcation and Chaos, vol. 10, no. 3, pp. 579-592, 2000.

[16] M. W. Spong, "Impact controllability of an air hockey puck," Systems and Control Letters, vol. 42, no. 5, pp. 333-345, 2001.
[17] L. Menini and A. Tornambe, "Control of (otherwise) uncontrollable linear mechanical systems through non-smooth impacts," Systems \& Control Letters, vol. 49, no. 4, pp. 311-322, 2003.

[18] M. Gerard and R. Sepulchre, "Stabilization through weak and occasional interactions: a billiard benchmark," in 6th IFAC-Symposium on Nonlinear Control Systems (NOLCOS), F. Allgower, Ed. Stuttgart, Germany: IFAC, 2004, pp. 75-80.

[19] B. Brogliato, M. Mabrouk, and A. Zavala-Rio, "On the controllability of linear juggling mechanical systems," Systems \& Control Letters, vol. 55, pp. 350-367, 2006.

[20] D. Sternad, M. Duarte, H. Katsumata, and S. Schaal, "Dynamics of a bouncing ball in human performance," Physical Review E, vol. 6301, no. 1, pp. art. no.-011 902, 2001.

[21] _ - "Bouncing a ball: tuning into dynamic stability," J Exp Psychol Hum Percept Perform, vol. 27, no. 5, pp. 1163-84, 2001.

[22] R. Ronsse, P. Lefevre, and R. Sepulchre, "Rhythmic feedback control of a blind planar juggler," IEEE Transactions on Robotics, submitted. 